\title{
Simplest driven conservative chaotic oscillator
}

\author{
H.P.W. Gottlieb ${ }^{\text {a }}$ J.C. Sprott ${ }^{\text {b }}$ \\ ${ }^{\text {a }}$ School of Science, Griffith University, Nathan, Queensland 4111, Australia \\ ${ }^{\mathrm{b}}$ Department of Physics, University of Wisconsin, Madison, WI 53706, USA
}

August 2001

Communicated by

\section{Abstract}

Sinusoidally-driven oscillator equations with a power-law nonlinearity are investigated computationally to determine the driving frequency which produces the "most chaos", i.e. the maximized largest Lyapunov exponent. It is argued that the "simplest" such driven chaotic oscillator has a cubic nonlinearity $x^{3}$.

PACS: 05.45.-a; 02.30.Hq

Keywords: Chaos; Non-autonomous; Differential equations; Lyapunov exponents 
The phenomenon of chaos has attracted widespread attention amongst mathematicians, physicists and engineers. Whilst many researchers analyze complicated, physically motivated configurations, there is also a need to investigate simple equations which may capture the essence of chaos in a less involved setting, thereby aiding the understanding of essential characteristics. A wide-ranging programme of searching for simple chaotic three-dimensional autonomous systems of differential equations, commenced by Sprott [1], and also for simple chaotic thirdorder ordinary differential equations ("jerk equations") instigated by Gottlieb [2], has been sustained by Sprott, Linz and others ([3], and references therein).

Nonautonomous, i.e. driven, systems are another widely studied class which provide a wealth of chaotic situations (e.g. [4] and references therein). This paper investigates algebraically simple examples of sinusoidally-driven conservative chaotic flows.

A "simple" equation should if possible have only one nonlinear term and only one essential control parameter. For driven systems, this constraint effectively limits the nonlinear term to being a power of the dependent variable $x$ or variants thereof, and precludes a damping term. Thus these flows will be conservative. In a general driven oscillator equation of the form $\ddot{x}+\alpha x^{p}=\beta \sin \omega t$, two of the three parameters $\alpha, \beta$, $\omega$ may be eliminated through a change of scales of $x$ and $t$. To standardize the equations, we shall always set the parameters $\alpha=1$ and $\beta=1$, and regard the driving angular frequency $\omega$ as the control parameter. Some earlier related investigations, such as those described in [4], set $\omega=1$, and regarded $\beta$ as the control parameter. Our standard equation, which preserves parity and allows for non-integer total powers $p$, will be 


$$
d^{2} x / d t^{2}+\operatorname{sgn}(x)|x|^{p}=\sin \omega t .
$$

The $\operatorname{sgn}(x)$ term makes the restoring force "centre-seeking" so that the trajectories are bounded. This equation is related to the "amplitude" equation

$$
d^{2} X / d T^{2}+\operatorname{sgn}(X)|X|^{p}=\beta \sin T
$$

by the dependent variable scale transformation $X=\omega^{2 /(1-p)} x$, and the independent variable scale transformation $T=\omega t$, with $\beta=\omega^{2 p /(1-p)}$. Conversely, $x=\beta^{-1 / p} X$ and $t=\beta^{(p-1) / 2 p} T$ with $\omega=\beta^{(1-p) / 2 p}$.

Lyapunov exponents are commonly used in the definition of dynamical chaos. It has been shown [5] that, under (invertible) transformations of the dependent variable, the Lyapunov exponents are invariant. However, since these exponents arise essentially in an exponential time dependence of the form $\exp (\lambda t)$, they obviously change if a time variable scale transformation is made, inversely as the scale. Thus the Lyapunov exponents $\Lambda$ for the above "amplitude" equation (2) and those of our standard "frequency" equation (1) are simply related by the relation $\lambda t=\Lambda T$, i.e. by $\Lambda$ $=\omega^{-1} \lambda$ or $\lambda=\beta^{(1-p) / 2 p} \Lambda$. Numerical experiments on the two equations can therefore be directly related. Moreover, chaos in one of the equations (1), (2) will be accompanied by chaos in the other, with corresponding largest positive Lyapunov exponents related as above. Lyapunov exponents here (base $e$ ) were computed using the method of Wolf et al. [6].

The simplest driven chaotic system would then be equation (1) with the lowest parity-conserving integer power $p=3$, which corresponds to the simplest nonlinear $\mathbf{C}^{\infty}$ function $x^{3}$ (i.e. having all derivatives continuous). This is the dissipationless limit of the Duffing oscillator which, including the damping, was investigated for instance by Ueda [7]. It would correspond physically to a mass attached to the middle of an 
initially unstretched vertical wire fixed at the ends and driven with a sinusoidal horizontal force [8]. A plot of the largest Lyapunov exponent versus the angular frequency parameter $\omega$ for equation (1) with $p=3$ (for initial conditions $x=d x / d t=0$ ) is given in Fig. 1. The "simplest, most chaotic" equation can then be found by searching for the value of control parameter $\omega$ which maximizes the largest Lyapunov exponent, for $p=3$. This exponent has value 0.097 , and occurs for $\omega=1.88$ in equation (1). A variant of simulated annealing was used to find this value. For this $p=$ 3, $\omega=1.88$ case, a Poincaré section in the $x-d x / d t$ plane for $\omega t \bmod 2 \pi=0$ for various initial conditions is depicted in Fig. 2. The prevalence of chaos is evident.

Other values of $p$ also produce interesting results. Some cases are difficult to optimize since there are rapid variations of Lyapunov exponent with frequency and the calculated Lyapunov exponent converges slowly. The case $p=5$ is shown in Fig. 3. Table 1 shows values of the maximized largest Lyapunov exponent and corresponding angular frequency for some representative values of $p$ (for initial conditions $x=d x / d t=0$ ). It appears that, for $p>1$, these quantities increase as $p$ increases. The largest Lyapunov exponent, if positive, for arbitrary $p$ and $\omega$ between 0 and 10 is shown on a grey scale in Fig. 4, for initial conditions $x=d x / d t=0$. In this plot $p$ is incremented in units of $1 / 64$ and $\omega$ is incremented in units of $1 / 48$. Note that for $p=1$ the field is white, corresponding to the conventional linear driven simple harmonic oscillator for which there is no chaos. An interesting feature is the increasingly chaotic behaviour $p$ approaches zero. Although $p$ is decreasing, the nonlinearity, as measured by the divergence from the linear value 1 , is actually increasing. Table 1 includes the results of computations for $p=1 / 2$. For the actual 
case $p=0$, corresponding to a sinusoidally driven oscillator with an intrinsic force $\operatorname{sign}(x)$, no chaotic solutions were found.

Numerical experiments also show that the form of the periodic drive is not critical. For example, a square wave of similar frequency also produces chaos in these systems.

It would be of interest to find the "most chaotic, simple" equation, by searching amongst the powers $p$ in equation (1) to find the equation and the corresponding value of $\omega$ with the greatest value for the maximized largest Lyapunov exponent. Such an equation, controlled by the single parameter $\omega$, could provide the clearest demonstration of chaos. However, as mentioned above, computations, as summarized in Table 1 and reflected in Fig. 4, indicate that the maximized largest Lyapunov exponent, for $p>1$, simply increases as $p$ increases. Such a search may therefore be illusory. The idea is nevertheless mentioned here, as it may be relevant to other classes of chaotic flows specified by other criteria.

Other, more complicated, nonlinearities may also be investigated to find parameter values that maximize the largest Lyapunov exponent. Some results may be found in [9]. 


\section{References}

[1] J.C. Sprott, Physics Letters A 173 (1993) 21-24.

[2] H.P.W. Gottlieb, American Journal of Physics 64 (1996) 525.

[3] J.C. Sprott, S.J. Linz, International Journal of Chaos Theory and Applications 5 (2000) 3-22.

[4] J.M.T. Thompson, H.B. Stewart, Nonlinear Dynamics and Chaos (Wiley, Chichester, 1987).

[5] R. Eichhorn, S.J. Linz, P. Hanggi, Chaos, Solitons and Fractals 12 (2001) 1377-1383.

[6] A. Wolf, J.B. Swift, H.L. Swinney, J.A. Vastano, Physica D 16 (1985) 285-317.

[7] Y. Ueda, in P.J. Holmes (ed.), New Approaches to Nonlinear Problems in Dynamics (SIAM, Philadelphia, 1980), pp. 311-322.

[8] R. E. Mickens, An Introduction to Nonlinear Oscillations (Cambridge Univ. Press, Cambridge, 1981), pp. 30 .

[9] J.C. Sprott, http://sprott.physics.wisc.edu/chaos/drivcons.htm 


\section{Figure and Table Captions.}

Fig.1. Largest Lyapunov exponent $\lambda$ versus driving angular frequency $\omega$ for equation (1) with $p=3$.

Fig. 2. Poincaré section in the $x$ - $d x / d t$ plane for $p=3, \omega=1.88$ case of equation (1), for $\omega t \bmod 2 \pi=0$.

Fig. 3. As for Figure 1, but with $p=5$.

Fig. 4. Largest positive Lyapunov exponent for arbitrary $p$ and $\omega$ in equation (1), shown on a grey scale - blacker tone for larger exponent.

Table 1. Maximized largest Lyapunov exponent $\lambda$ and corresponding angular frequency $\omega$ for equations of the type (1) for various $p$. (Initial conditions $x=d x / d t=$ 0.) 
Fig.1. Largest Lyapunov exponent $\lambda$ vs driving angular frequency $\omega$ for equation (1) with $p=3$.

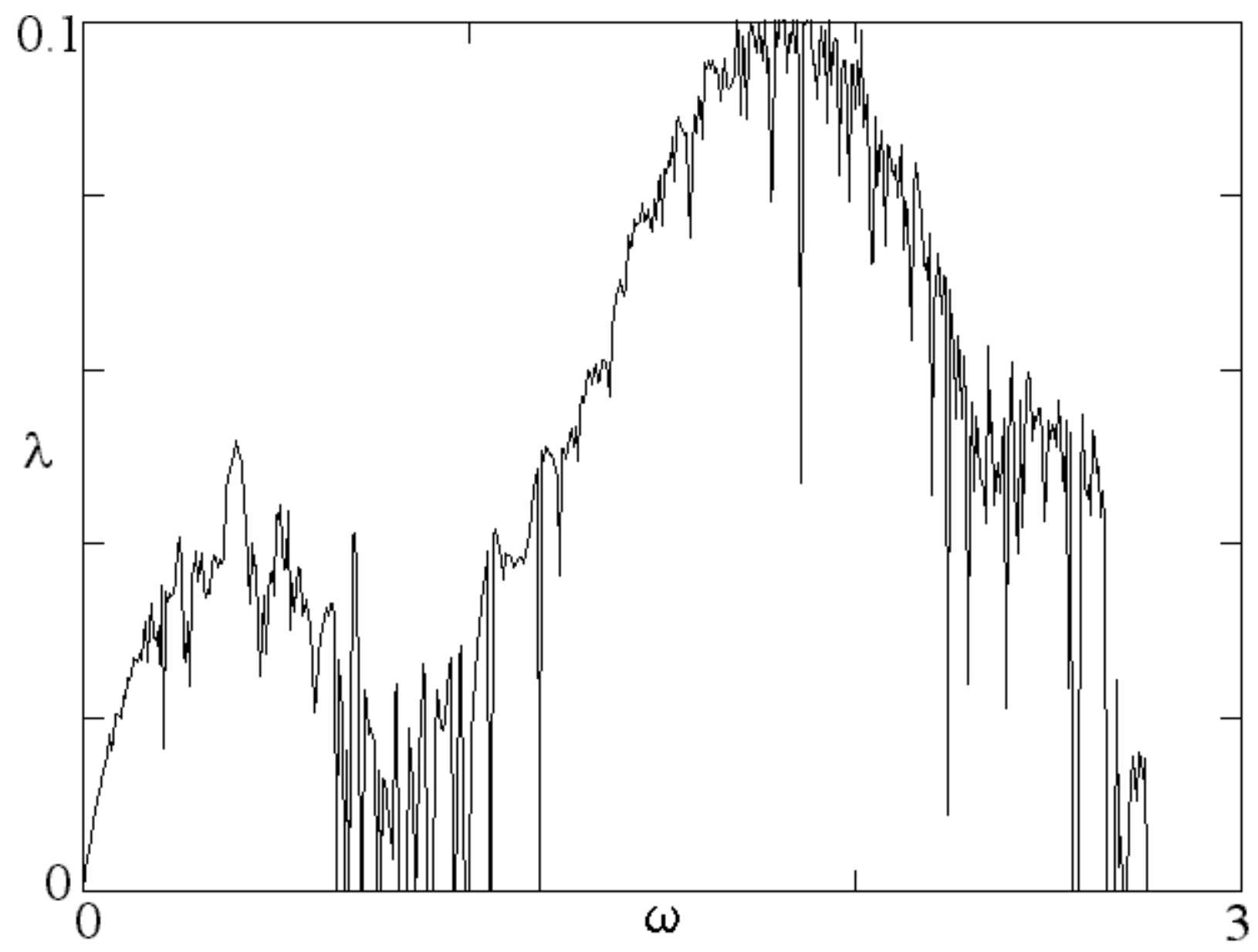


Fig. 2. Poincaré section in $x-d x / d t$ plane for $p=3, \omega=1.88$ case of equation (1), for $\omega t$ $\bmod 2 \pi=0$.

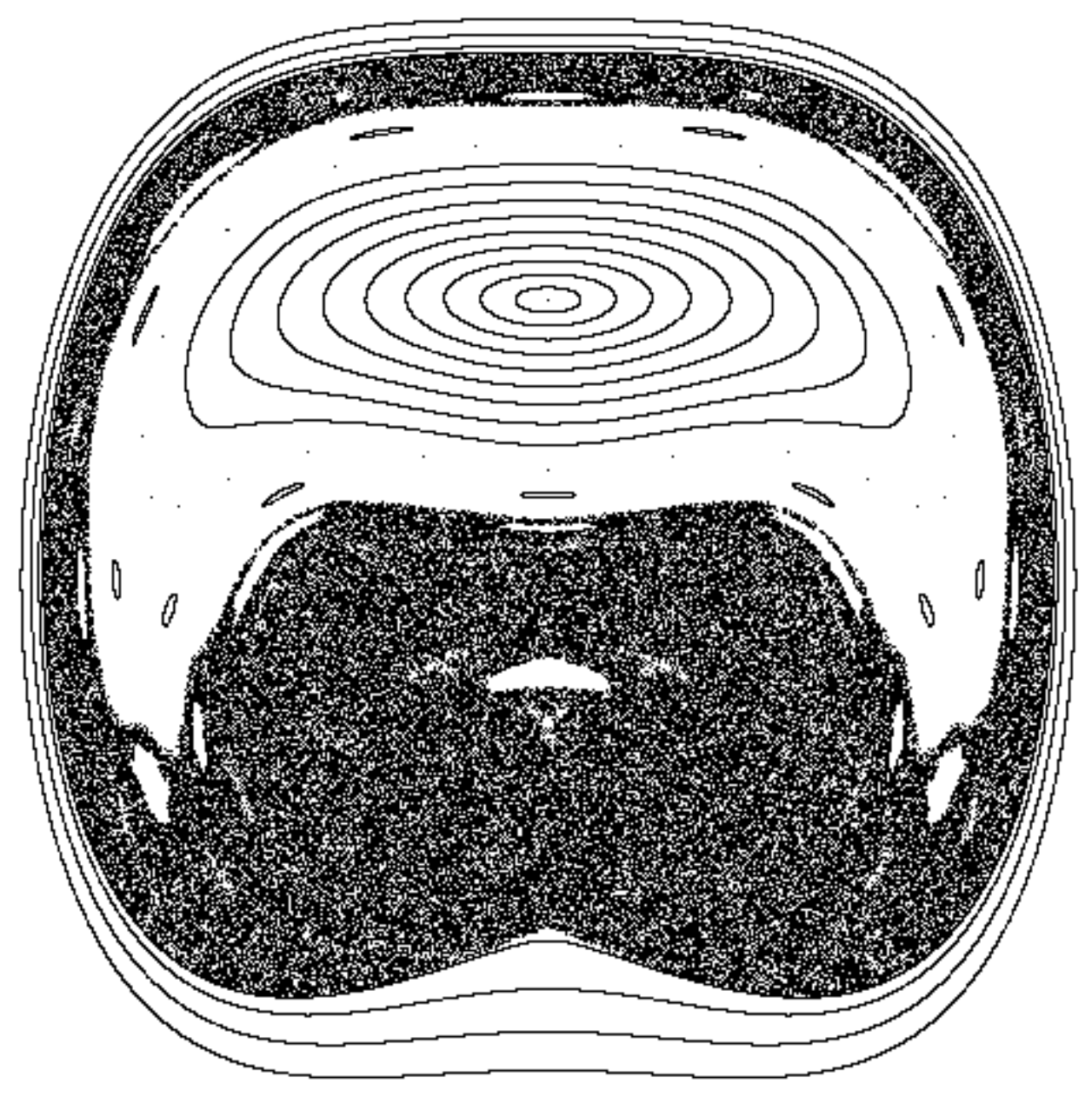


Fig. 3. As for Figure 1, but with $p=5$.

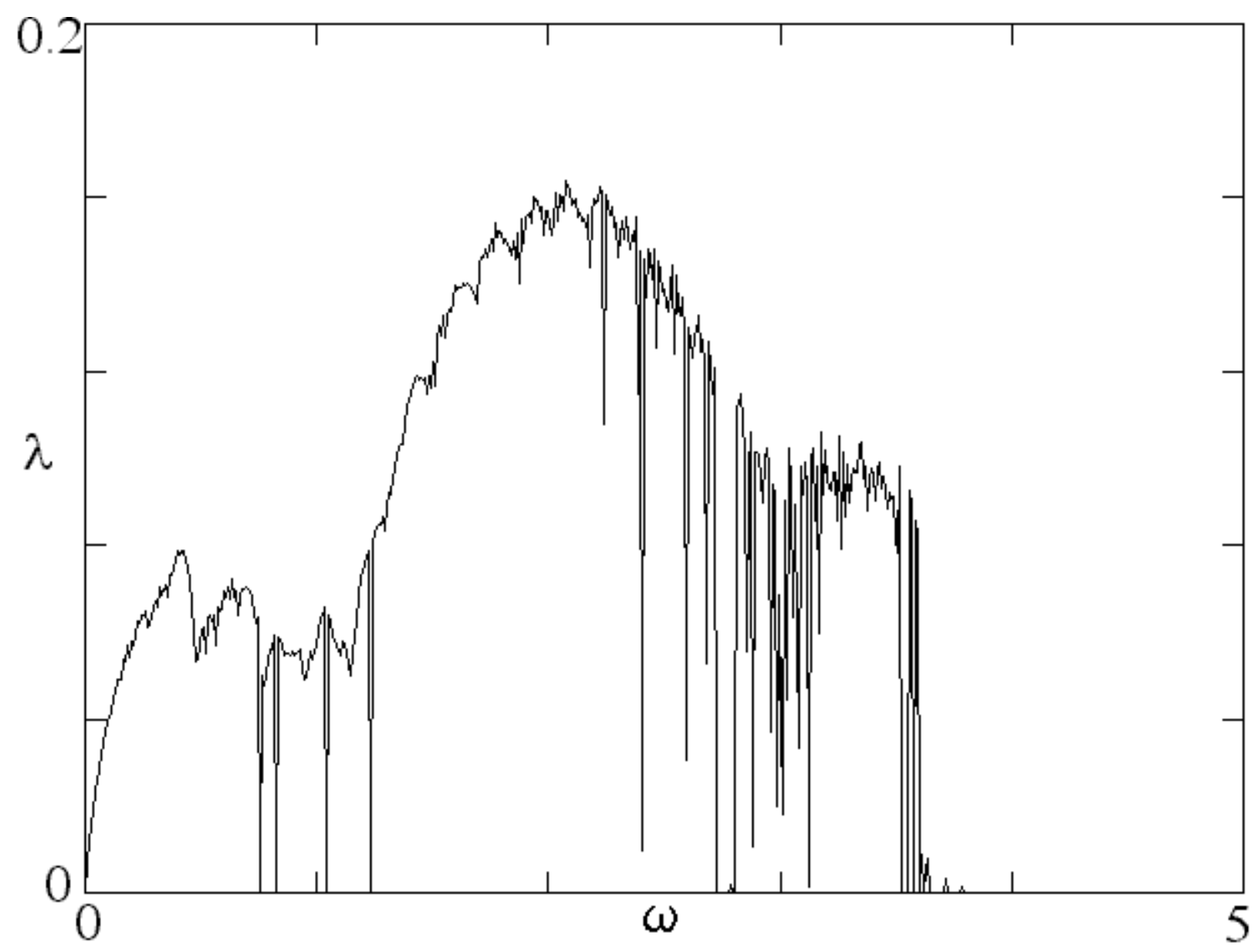


Fig.4. Largest positive Lyapunov exponent for arbitrary $p$ and $\omega$ in equation (1), shown on a grey scale - blacker tone for larger exponent.

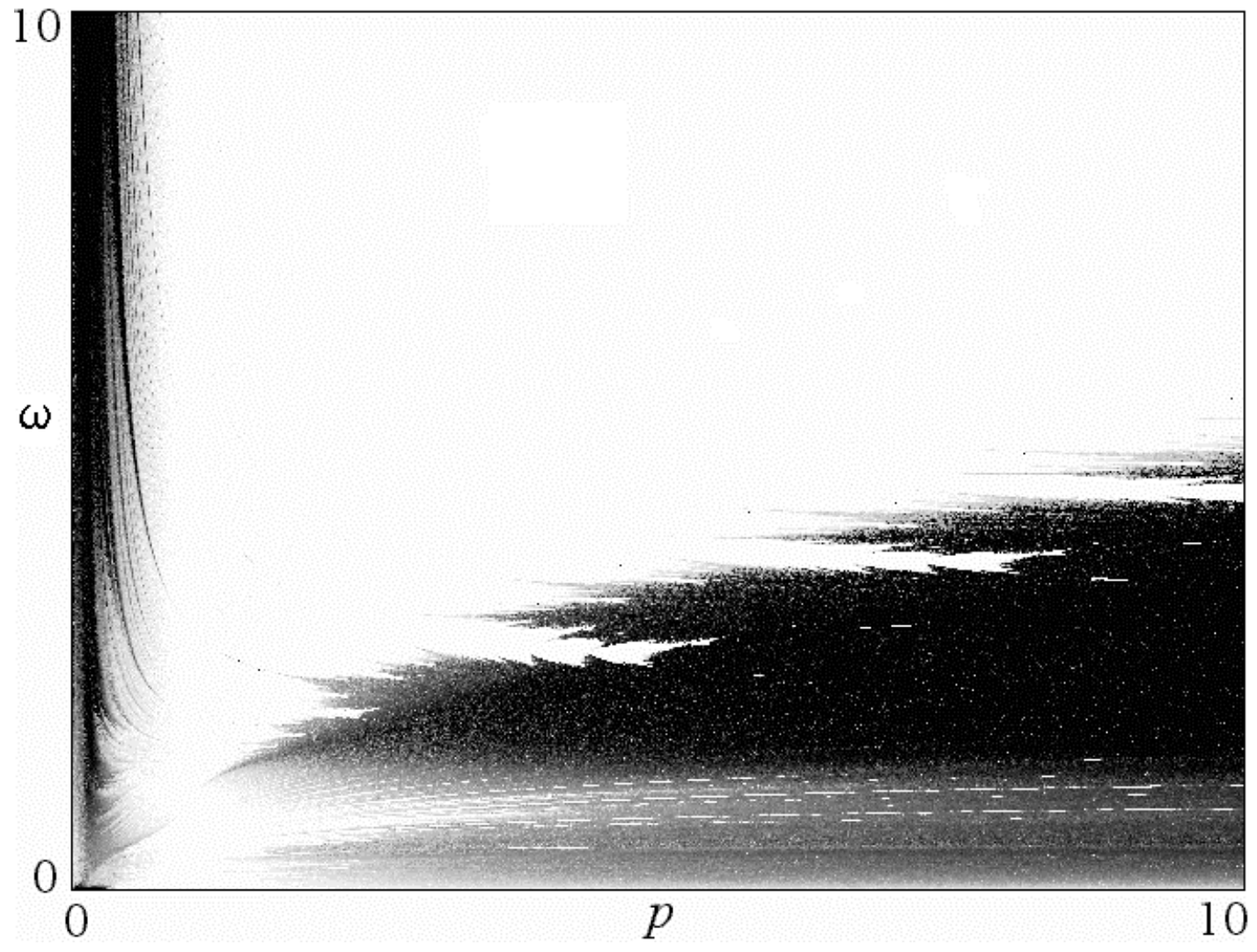


Table 1. Maximized largest Lyapunov exponent $\lambda$ and corresponding angular frequency parameter $\omega$ for equations of the type (1) for various $p$. (Initial conditions $x$ $=d x / d t=0$.

\begin{tabular}{lll}
$p$ & $\lambda$ & $\omega$ \\
& & \\
& & \\
$1 / 2$ & 0.123 & 5.57 \\
2 & 0.051 & 1.61 \\
3 & 0.097 & 1.88 \\
4 & 0.139 & 2.00 \\
5 & 0.163 & 2.19 \\
7 & 0.198 & 2.32 \\
9 & 0.230 & 2.58 \\
11 & 0.242 & 2.79 \\
\hline & & \\
\hline
\end{tabular}

